\title{
Running Spectral Index from Inflation with Modulations
}

\author{
Takeshi Kobayashi ${ }^{1}$, and Fuminobu Takahashi ${ }^{2, \dagger}$ \\ 1 Institute for Cosmic Ray Research, The University of Tokyo, \\ 5-1-5 Kashiwanoha, Kashiwa, Chiba 277-8582, Japan \\ 2 Institute for the Physics and Mathematics of the Universe, \\ The University of Tokyo, 5-1-5 Kashiwanoha, Kashiwa, Chiba 277-8583, Japan
}

\begin{abstract}
We argue that a large negative running spectral index, if confirmed, might suggest that there are abundant structures in the inflaton potential, which result in a fairly large (both positive and negative) running of the spectral index at all scales. It is shown that the center value of the running spectral index suggested by the recent CMB data can be easily explained by an inflaton potential with superimposed periodic oscillations. In contrast to cases with constant running, the perturbation spectrum is enhanced at small scales, due to the repeated modulations. We mention that such features at small scales may be seen by $21 \mathrm{~cm}$ observations in the future.
\end{abstract}

\section{INTRODUCTION}

Cosmic inflation [1] sets the initial conditions for the subsequent Hot Big Bang cosmology, and also generates the primordial curvature perturbation of the universe. The nature of this primordial density perturbation is studied through various cosmological observations, and especially CMB data can be well fitted by a power-law primordial spectrum, which is a typical prediction of the standard slow-roll inflation.

Interestingly, however, when relaxing the assumption of a power-law spectrum, latest CMB observations show a slight preference for a fairly large negative running spectral index. Ignoring tensor modes, the WMAP 7-yr data combined with ACT 2008 data give the bounds $n_{s}=$ $1.032 \pm 0.039$ and $d n_{s} / d \ln k=-0.034 \pm 0.018(68 \%$ CL) at the pivot scale $k_{0}=0.002 \mathrm{Mpc}^{-1}$ 4. Even with the tensor modes, similar bounds are obtained using the WMAP data only [5]. In the usual slow-roll inflation model with a featureless potential, the running spectral index is second order in the slow-roll parameters, and so it is expected to be of $O\left(10^{-3}\right)$. Thus, although the evidence for the running spectral index is not conclusive, it is worth studying its implications for the inflation models.

The center value of the running spectral index, if taken at face value, suggests either the inflaton potential with a step-like feature (see e.g. 6 6-11] and references therein), or multiple inflation [12, 13], since otherwise inflation would terminate within about 30 e-foldings [14. In both cases, fine-tuning is needed to ensure that the CMB scale exits the horizon at a specific time when the inflaton comes close to the feature in its potential, or at about 10 e-foldings before the end of the first inflation. Such fine-tuning seems a puzzle and calls for explanation.

We argue in this paper that, if there is indeed large negative running spectral index, this might suggest that the inflaton potential has structures everywhere in the po-

*takeshi.kobayashi@ipmu.jp

tfuminobu.takahashi@ipmu.jp tential and such a potential may be common in the landscape of inflation models. Such repeated modulations to the inflaton potential can account for a perturbation spectrum which is blue tilted with negative running at the CMB scale, if one period of the modulations is large enough to encompass the observed scales. Inflaton potentials with modulations can lead to repeated features in the perturbation spectrum, in contrast to the case of single-stepped potentials. See also [15] for related work in the context of Natural Inflation models [16, 17] with extra dimensions [18, 19.

Modulations to the inflaton potential can arise due to the microphysics governing inflation. Especially for large-field models, one expects that the super-Planckian field ranges may contain many features whose scale is typically related to that of the underlying microphysics. Explicit constructions of large-field models in string theory 20 22 actually show that periodic modulations of the potential can show up, whose period is determined by e.g., the size of the internal compactified space. (See also [23, 24] for discussions on small-scale features along the inflaton field range in the cosmic landscape.)

To be explicit, we consider large-field inflation from power-law potentials with superimposed periodic modulations, and show that the center value of the running spectral index can be realized. What differs from the previous studies using single-stepped potentials is that the running spectral index is not localized, but it is negative at the entire CMB scales and becomes positive at small scales. Such characteristic behavior of the density perturbation may be seen by the future $21 \mathrm{~cm}$ observations [25, 26]. The potential detection of such oscillatory feature in the power spectrum by future observations was studied in Ref. [27, with the main focus on oscillations with (much) shorter period.

The rest of the paper is organized as follows. In Sec. II we briefly explain our idea of generating the running spectral index. Then we estimate the power spectrum of the density perturbation based on inflation models with a linear, and then a generalized polynomial potential superimposed with periodic modulations, both analytically and numerically in Sec. III and Sec. IV. The last section 
is devoted to discussions and conclusions.

\section{BASIC IDEA}

Let us first give our basic idea in this section. In this paper we use the units $c=\hbar=M_{p}=1$.

The fact that the CMB data can be well fitted by a Gaussian power-law primordial spectrum indicates that the standard slow-roll inflation paradigm is a valid description of the evolution of the early universe. However, if one looks at the details of the inflaton potential, there might be non-trivial structures which do not change the overall behavior of the inflaton, but nonetheless affect the tilt and/or the running spectral index in a non-negligible way.

If the running of the spectral index is large at the $\mathrm{CMB}$ scales, it may suggest that the inflaton potential has steplike structures everywhere, since severe fine-tuning would be needed otherwise. So we consider an inflaton potential $V(\phi)$ with modulations such as shown in Fig. 1. We decompose the potential as

$$
V(\phi)=V_{0}(\phi)+V_{\text {mod }}(\phi),
$$

where the first term is a smooth potential sourcing a (nearly) constant spectral tilt, and the second term represents the modulations. We assume that the overall inflaton dynamics (such as the Hubble parameter and the inflaton velocity) is not altered drastically by the modulations, i.e.,

$$
\begin{aligned}
& \left|V_{0}(\phi)\right| \gg\left|V_{\text {mod }}(\phi)\right|, \\
& \left|V_{0}^{\prime}(\phi)\right|>\left|V_{\text {mod }}^{\prime}(\phi)\right| .
\end{aligned}
$$

We further suppose that the slow-roll approximations

$$
3 H \dot{\phi} \simeq-V^{\prime}, \quad 3 H^{2} \simeq V,
$$

are valid. Then, in order for the running of the spectral index to be large enough so that the spectral tilt switches between blue and red within the observed scales, we require

$$
\begin{aligned}
\left|V_{0}^{\prime \prime}(\phi)\right| & \lesssim\left|V_{\text {mod }}^{\prime \prime}(\phi)\right|, \\
\left|V_{0}^{\prime \prime \prime}(\phi)\right| & \ll\left|V_{\text {mod }}^{\prime \prime \prime}(\phi)\right| .
\end{aligned}
$$

Thus, we are invoking a hierarchy

$$
\left|\frac{V_{m o d}}{V_{0}}\right| \ll\left|\frac{V_{m o d}^{\prime}}{V_{0}^{\prime}}\right| \ll\left|\frac{V_{m o d}^{\prime \prime}}{V_{0}^{\prime \prime}}\right| \ll\left|\frac{V_{m o d}^{\prime \prime \prime}}{V_{0}^{\prime \prime \prime}}\right| .
$$

We note that the conditions (5), (6), and (7) need not hold all along the inflaton potential, but are only required to be satisfied occasionally during inflation for large running of the spectral index to be produced.

An important assumption here is that the effect of the modulations $V_{\text {mod }}$ (and its derivatives) on the inflaton dynamics is sufficiently small when averaged over a sufficiently long time or large field space. One example for

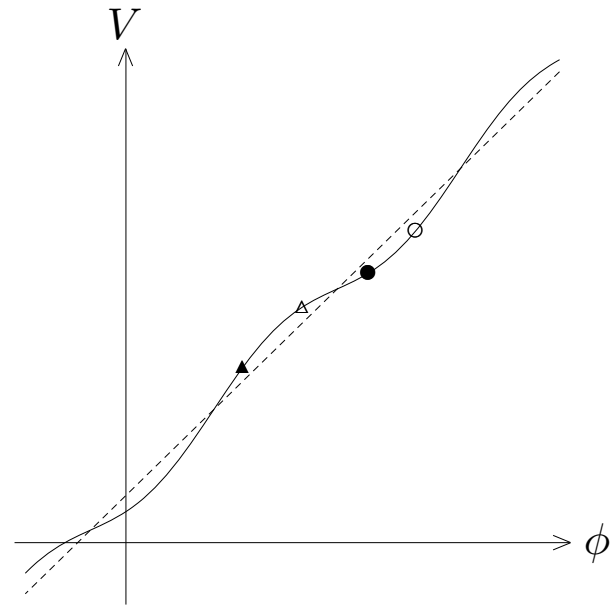

FIG. 1: A sketch of the inflaton potential with superimposed periodic oscillations. The dashed line is a smooth potential without modulations. The modulations are amplified for visualization purposes. The resulting curvature perturbation and its tilt at scales exiting the horizon as the inflaton approaches the markers are shown in Fig. 2 and Fig. 3 The observed CMB scales are considered to lie within a half oscillation period.

such modulations is a sine function, and we will see in the following sections that the above conditions can be easily satisfied in this case. We emphasize here that the effect of modulations can be missed in such an analysis that the inflaton potential is expanded in terms of a power of $\phi$ up to a finite order [14]. On the other hand, as we shall see, the spectral index and its running are easily modified by the modulations, while not affecting the overall inflaton dynamics.

The spectrum of the curvature perturbation can be computed,

$$
P_{\zeta} \simeq \frac{H^{4}}{4 \pi^{2} \dot{\phi}^{2}},
$$

which should be estimated at the time of horizon exit $k=$ $a H$. Note that, since $\dot{\phi}$ is determined by $V^{\prime}$, the main contribution to the power spectrum arises from the smooth part $V_{0}^{\prime}$, and that the $V_{m o d}^{\prime}$ results in small oscillations of the perturbation spectrum as illustrated in Fig. 2. The oscillatory feature of the perturbation spectrum arises mainly from $\dot{\phi}$ (i.e. $V_{m o d}^{\prime}$ ), and the modulations of $H$ (i.e. $V_{\text {mod }}$ ) is subdominant in the following analysis. The spectrum is blue (red) for scales exiting the horizon when the inflaton is rolling along the potential whose curvature is positive (negative). To be precise, since the dropping of the Hubble parameter reddens the perturbation spectrum, the resulting spectrum becomes blue tilted when the deceleration of the inflaton (i.e. the positive curvature of the potential) is large enough.

Before computing the tilt of the spectrum, let us pause and comment on the validity of the slow-roll approximations. Expressions for the spectral index and its running (and its running, and so on) derived using the slow-roll 


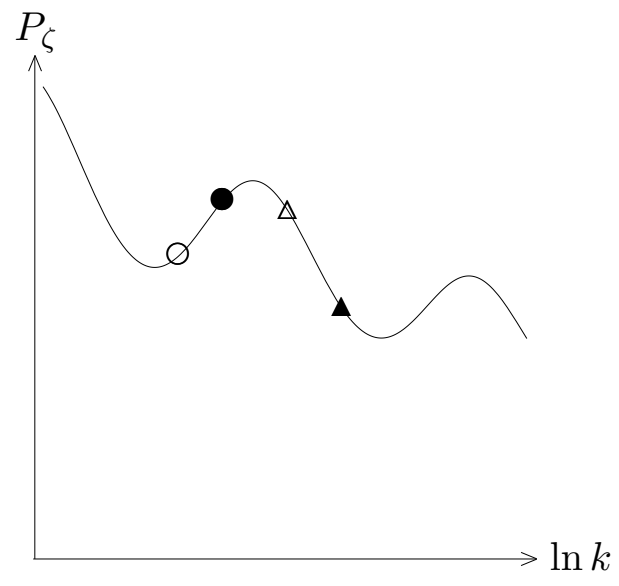

FIG. 2: The curvature perturbation spectrum generated from the inflaton potential in Fig. 1 .

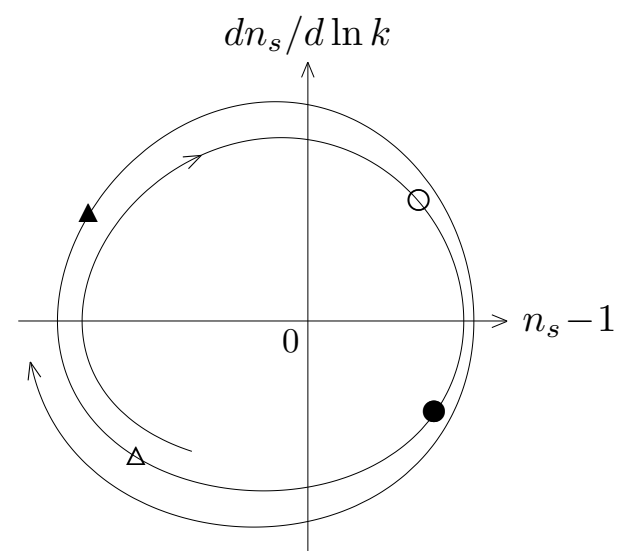

FIG. 3: Spectral index and its running from the inflaton potential in Fig. 1 .

approximations contain errors of order of the approximate results multiplied by

$$
\frac{1}{H} \frac{d}{d t} \simeq-\frac{V^{\prime}}{V} \frac{d}{d \phi}
$$

Therefore, as long as this is smaller than unity, results obtained using the slow-roll approximations should be good estimates. Such condition is satisfied for the explicit cases we study later on, since we require the observed scales to be generated within a half oscillation period. For detailed analyses of inflation and primordial perturbations beyond the slow-roll approximations, see e.g. 28 35].

Then the spectral index and its running can be estimated as follows,

$$
\begin{aligned}
n_{s}-1 & =\frac{d \ln P_{\zeta}}{d \ln k} \simeq-6 \epsilon+2 \eta \\
& \simeq-6 \epsilon_{0}+2 \eta_{0}-6 \frac{V_{0}^{\prime} V_{m o d}^{\prime}}{V_{0}^{2}}+2 \frac{V_{m o d}^{\prime \prime}}{V_{0}}
\end{aligned}
$$

$$
\begin{aligned}
\frac{d n_{s}}{d \ln k} \simeq & -24 \epsilon^{2}+16 \epsilon \eta-2 \xi \\
\simeq & -24 \epsilon_{0}^{2}+16 \epsilon_{0} \eta_{0}-2 \xi_{0} \\
& -24 \frac{V_{0}^{\prime 3} V_{m o d}^{\prime}}{V_{0}^{4}}+8 \frac{V_{0}^{\prime 2} V_{m o d}^{\prime \prime}}{V_{0}^{3}}-2 \frac{V_{0}^{\prime} V_{m o d}^{\prime \prime \prime}}{V_{0}^{2}},
\end{aligned}
$$

where

$$
\epsilon \equiv \frac{1}{2}\left(\frac{V^{\prime}}{V}\right)^{2}, \eta \equiv \frac{V^{\prime \prime}}{V}, \xi \equiv \frac{V^{\prime} V^{\prime \prime \prime}}{V^{2}} .
$$

In the final expressions, we have kept contributions from the smooth potential $V_{0}$ which are denoted by parameters with the subscript 0 , and also the leading oscillatory terms due to the modulations $V_{\text {mod }}$, by using the hierarchy (7) and assuming that the inequality (3) is well satisfied. When the oscillatory terms are dominant, the tilt of the perturbation spectrum repeatedly switches between red and blue, hence the spectral index $n_{s}-1$ as well as the running $d n_{s} / d \ln k$ change between positive and negative values. This results in a clockwise motion in the $\left(n_{s}-1\right)-d n_{s} / d \ln k$ plane as inflation proceeds, as illustrated in Fig. 3. Given that the pivot scale exited the horizon when the inflaton was around the filled circle in Fig. 1. in one of the repeated modulations, a blue tilted perturbation spectrum with negative running is obtained.

The hierarchy (7) implies that even if the modulations are so small that their effects on the Hubble parameter and the inflaton velocity are subleading, they can still significantly contribute to the spectral tilt of the perturbation spectrum. However, one should also note that such hierarchy can lead to the breakdown of the conditions (2) and (3) at other places along the inflaton potential, thus considerably affecting the inflaton dynamics in the end. We will see in the following sections using explicit examples that oscillatory modulations can satisfy the above conditions all through (or at least for a large fraction of) the inflationary period.

\section{LINEAR POTENTIAL WITH PERIODIC OSCILLATIONS}

Now let us consider an explicit inflation model. As a simple example, we consider curvature perturbations from a linear inflaton potential [21, 36] with superimposed periodic oscillations [22, 37] (cf. Fig. 1):

$$
V(\phi)=\lambda \phi+\Lambda^{4} \cos \left(\frac{\phi}{f}+\theta\right),
$$

where $\lambda, \Lambda, f$, and $\theta$ are constants. The period of the oscillations is determined by $f$, which we assume to be sub-Planckian, i.e. $f<1$. This is often the case in explicit microscopic constructions, and is also compatible with our requirement that half of an oscillatory period roughly corresponds to the time duration when the observed scales exited the horizon. We also assume $V^{\prime}>0$, 
namely,

$$
\frac{\Lambda^{4}}{\lambda f}<1
$$

which guarantees that the inflaton is not trapped by the modulations during inflation. This condition is equivalent to (3).

Assuming that the slow-roll approximations (4) are valid, the spectrum of the curvature perturbation can be computed,

$$
P_{\zeta} \simeq \frac{\lambda \phi^{3}}{12 \pi^{2}}\left(1-\frac{\Lambda^{4}}{\lambda f} \sin \left(\frac{\phi}{f}+\theta\right)\right)^{-2},
$$

which should be estimated at the time of horizon exit. Note that in obtaining the right hand side we have omitted modulations of $H$ (i.e. $V$ ) which is subdominant due to (14) and $f \ll \phi$ (since inflation occurs for superPlanckian $\phi$ ).

Let us now check whether we can use expressions for the spectral tilt derived using the slow-roll approximations. (See also discussions below (9p.) Upon its computations, one finds

$$
\left|-\frac{V^{\prime}}{V} \frac{d}{d \phi}\right| \lesssim \frac{1}{\phi f}
$$

where the $f$ in the denominator of the right hand side comes from differentiating the oscillatory part. As we will soon see, in our case $(\phi f)^{-1}$ is smaller than unity, at least until when the CMB scale exits the horizon, due to the requirement that the period of the oscillations be large enough to encompass the observed scales. Therefore we trust results derived using the slow-roll approximations.

Then the spectral index and its running can be estimated as follows,

$$
\begin{aligned}
& n_{s}-1 \simeq-\frac{1}{\phi^{2}}\left(3+\frac{2 \Lambda^{4} \phi}{\lambda f^{2}} \cos \left(\frac{\phi}{f}+\theta\right)\right), \\
& \frac{d n_{s}}{d \ln k} \simeq-\frac{1}{\phi^{4}}\left(6+\frac{2 \Lambda^{4} \phi^{2}}{\lambda f^{3}} \sin \left(\frac{\phi}{f}+\theta\right)\right) .
\end{aligned}
$$

In the final expressions (17) and (18), we have only kept the leading oscillatory terms. One clearly sees that the spectral index and its running oscillate with growing oscillation amplitudes as inflation proceeds and $\phi$ becomes smaller. (The relative amplitude compared to the nonoscillatory part becomes smaller.) Note that the power of $f(<1)$ appearing in Eqs. (17) and (18) are larger than that of Eq. (15), and that is why the spectral index and its running can be significantly modified without affecting the power spectrum too much. One can easily check that the conditions (2) - (6) can be satisfied for a certain choice of $f$.

Let us now set the parameters of the potential so that the spectral index and its running at the pivot scale re- alizes the central values of the bounds from WMAP 7yr [5]:

$$
\begin{aligned}
n_{s} & =1.076 \pm 0.065, \\
d n_{s} / d \ln k & =-0.048 \pm 0.029,
\end{aligned}
$$

(both at $68 \% \mathrm{CL}$ ) when both the running and the tensor mode perturbations are allowed to vary, in addition to the spectral index. (The bound on the tensor-to-scalar ratio here is $r<0.49(95 \% \mathrm{CL})$ ). ) Note that the predicted power spectrum is not exactly the one used in the analysis by the WMAP team. However, since the period of oscillations is sufficiently long compared to the observed CMB scales, such a difference will not significantly change the likelihood distribution. For simplicity we use the above values in the following analysis.

We set the pivot scale to have exited the horizon when the inflaton field was at around $\phi_{0}=10$, which corresponds to about 50 e-folds before inflation ended. For a linear potential (without modulations), the number of e-folds obtained within the field range $\Delta \phi$ starting from $\phi_{0}$ is $\Delta \mathcal{N} \simeq \phi_{0} \Delta \phi$. Here we set $f$ by choosing the half period of oscillation from the pivot scale to correspond to about $\ln l_{\text {max }}^{\mathrm{CMB}} \simeq 7.5$ e-foldings with $l_{\text {max }}^{\mathrm{CMB}} \sim 2000$, i.e. $\pi f=\Delta \mathcal{N} / 2 \phi_{0}=0.75$. (Note that requiring the observed scales to lie within one oscillation period, i.e. $\Delta \mathcal{N} \gtrsim 10$, leads to $\left(\phi_{0} f\right)^{-1}<1$ which validates the analytic expressions derived using slow-roll approximations, as was discussed below $(9)$ and $\left.(16) .{ }^{1}\right)$ Then the remaining parameters $\lambda, \Lambda, \theta$ are determined so that the perturbation spectrum at the pivot scale realizes the COBE normalization 38] $P_{\zeta}=2.43 \times 10^{-9}$, as well as the central values of 191 . We choose $\lambda \approx 1.99 \times 10^{-10}, \Lambda \approx 1.77 \times 10^{-3}$, $\theta \approx-2.03$, and the inflaton field value when the pivot scale exits the horizon as $\phi \approx 9.98$. We note that the modulations' amplitude $\Lambda$ is of order the GUT scale.

With these parameter sets, we have numerically solved the equation of motion of the inflaton and the Friedmann equation, and computed the curvature perturbation spec$\operatorname{trum} P_{\zeta}=\left.\frac{H^{4}}{4 \pi^{2} \dot{\phi}^{2}}\right|_{k=a H}$, which is shown in Fig. 4. Oscillatory features are clearly seen in the spectrum. For comparison, we also plotted a spectrum with constant running $\alpha$,

$$
P_{\zeta}(k)=P_{\zeta}\left(k_{0}\right)\left(\frac{k}{k_{0}}\right)^{n_{s}\left(k_{0}\right)-1+\frac{1}{2} \alpha \ln \frac{k}{k_{0}}},
$$

extrapolated from values at the pivot scale $k_{0}=$ $0.002 \mathrm{Mpc}^{-1}$. The CMB observations probe scales from $k \sim 10^{-4} \mathrm{Mpc}^{-1}$ down to $\sim 0.1 \mathrm{Mpc}^{-1}$, and within this range these two lines are almost degenerate. The difference becomes evident only at small scales $k \gtrsim 10 \mathrm{Mpc}^{-1}$, which may be probed by the future proposal of the $21 \mathrm{~cm}$

\footnotetext{
1 The condition $(\phi f)^{-1}<1$ is satisfied until about 10 e-foldings before the end of inflation.
} 


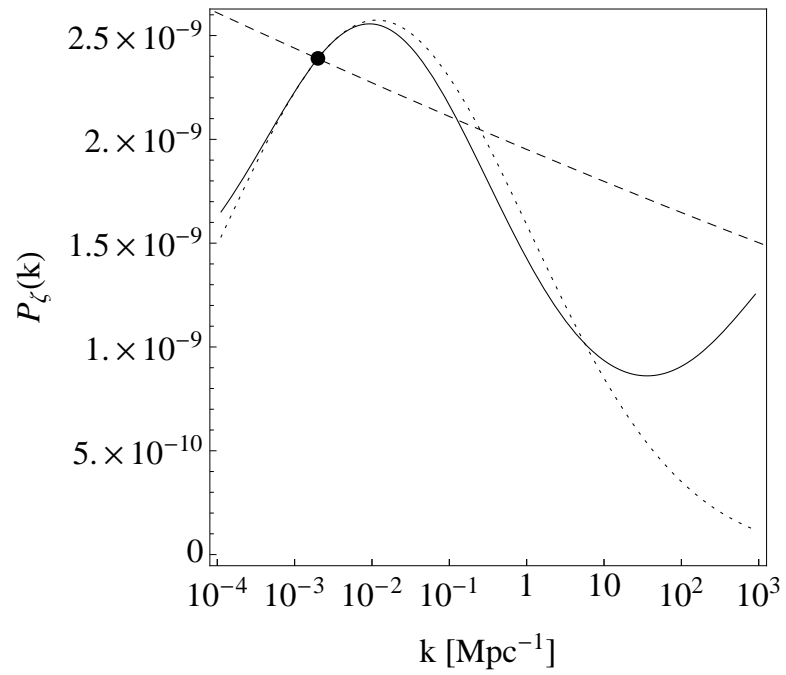

FIG. 4: Curvature perturbation spectrum (black solid line) from the inflaton potential (13), realizing central values of $\sqrt{19}$ at the pivot scale $k_{0}=0.002 \mathrm{Mpc}^{-1}$ which is denoted by the filled circle. Spectrum with constant running 20 extrapolated from values at the pivot scale is shown as the black dotted line. Spectrum from a linear potential without any modulation is shown as the black dashed line for comparison.

observations [25, 26]. We also plot the varying spectral index and its running in Fig. 5. For each oscillation in the inflaton potential, the trajectory in the phase space passes through the WMAP constrained region. As inflation proceeds, i.e. towards smaller scales, the radius of the orbit increases and also its center shifts towards smaller $n_{s}$.

\section{POWER-LAW POTENTIAL WITH PERIODIC OSCILLATIONS}

All the previous discussions can be straightforwardly extended to large-field inflation with general power-law potential (see e.g. [15, 40, 41] for models which exhibit quadratic inflaton potentials with superimposed periodic oscillations),

$$
V(\phi)=\lambda \phi^{n}+\Lambda^{4} \cos \left(\frac{\phi}{f}+\theta\right) .
$$

The condition equivalent to [14, which guarantees $V^{\prime}>$ 0 is now

$$
\frac{\Lambda^{4}}{n \lambda \phi^{n-1} f}<1
$$

but one should note that for $n>1$, this may break down as $\phi$ becomes smaller and the inflaton can be trapped in a local minimum due to the modulations.

The approximate expressions for the perturbation spectrum and its spectral tilt can be derived in a sim-

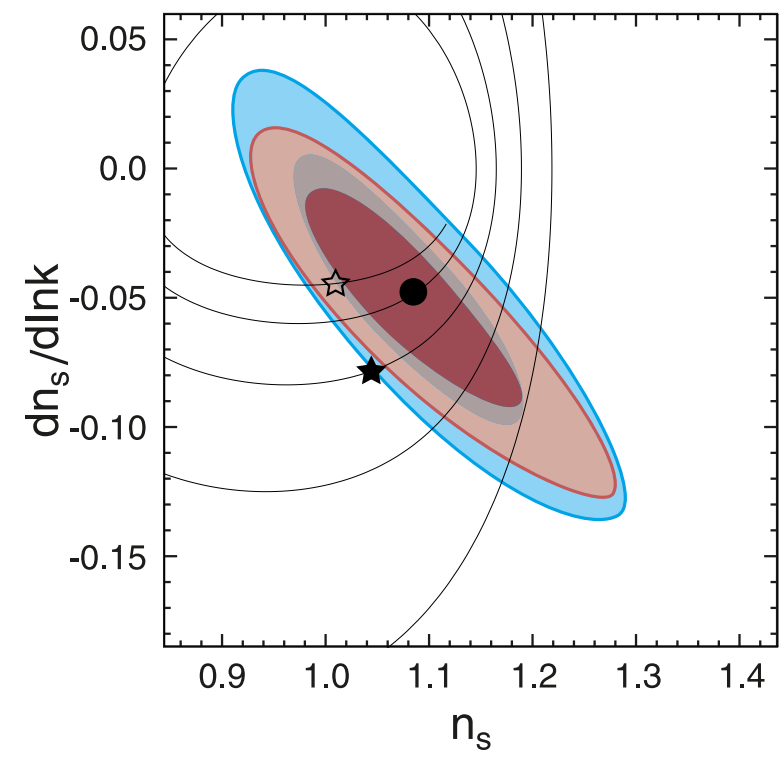

FIG. 5: Variation of the spectral index $n_{s}$ and its running $d n_{s} / d \ln k$ as inflation proceeds along the modulated potential (13). The plot starts from 70 e-folds before the end of inflation (end point of the innermost orbit), up to $\sim 10$ efolds before the end of inflation where most part of the orbit goes beyond the plotted region. Filled circle corresponds to the pivot scale (about 50 e-folds before the end of inflation). The scale which exited the horizon 15 e-folds before (after) the pivot scale is indicated by an open (filled) star. Superimposed contours are constraints from 5-year WMAP (blue) and from 5-year WMAP+BAO+SN (red) (68\% and 95\% CL), when tensor moder perturbations, spectral tilt, and running index are allowed to vary 39.

ilar fashion,

$$
\begin{aligned}
& P_{\zeta} \simeq \frac{\lambda \phi^{n+2}}{12 \pi^{2} n^{2}}\left(1-\frac{\Lambda^{4}}{n \lambda f \phi^{n-1}} \sin \left(\frac{\phi}{f}+\theta\right)\right)^{-2}, \\
& n_{s}-1 \\
& \quad \simeq-\frac{1}{\phi^{2}}\left(n(n+2)+\frac{2 \Lambda^{4}}{\lambda f^{2} \phi^{n-2}} \cos \left(\frac{\phi}{f}+\theta\right)\right), \\
& \frac{d n_{s}}{d \ln k} \\
& \simeq-2 n \frac{1}{\phi^{4}}\left(n(n+2)+\frac{\Lambda^{4}}{\lambda f^{3} \phi^{n-3}} \sin \left(\frac{\phi}{f}+\theta\right)\right) .
\end{aligned}
$$

One can see that the spreading of the oscillation amplitudes of $n_{s}-1$ and $d n_{s} / d \ln k$ as inflation proceeds is more drastic for larger $n$, since oscillations with constant amplitude as in (21) become relatively more significant at small $\phi$. For comparison, we have plotted oscillating trajectories in the $\left(n_{s}-1\right)-d n_{s} / d \ln k$ plane for the linear $(n=1)$ and quadratic $(n=2)$ potentials with modulations in Fig. 6. The parameters of the potentials are chosen in order to realize the central values of $(19)$ and the 


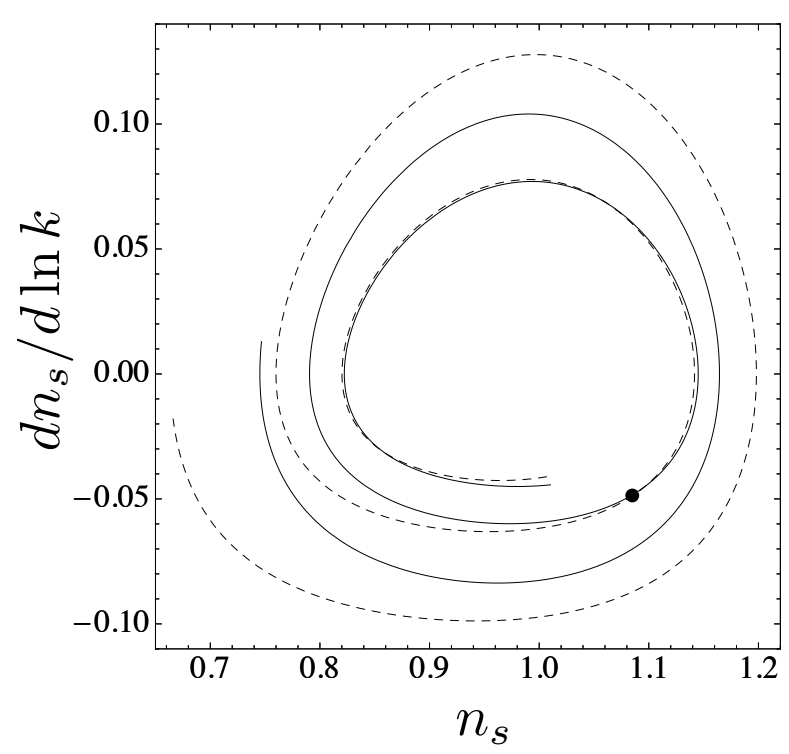

FIG. 6: Variation of the spectral index $n_{s}$ and its running $d n_{s} / \ln k$ for linear (solid) and quadratic (dashed) potentials with modulations. Filled circle indicates when the pivot scale exited the horizon. Both trajectories are plotted from 15 e-folds before the pivot scale exited the horizon (end points of the innermost orbits), up to 20 e-folds after the pivot scale (end points of the outermost orbits).

COBE normalization at the pivot scale. The case with the linear potential was discussed in the previous section. For the quadratic potential, the pivot scale is set to have exited the horizon at around $\phi_{0}=\sqrt{200}$, and $f$ is chosen to realize a half oscillation period lasting for about 7.5 e-foldings from the pivot scale, i.e. $\pi f=\Delta \mathcal{N} / \phi_{0} \approx 1.06$. The remaining parameters are chosen as $\lambda \approx 1.95 \times 10^{-11}$, $\Lambda \approx 2.52 \times 10^{-3}, \theta \approx-2.06$, and the inflaton field value when the pivot scale exits the horizon as $\phi \approx 14.1 .^{2}$

Normalized at the pivot scale, one sees that the trajectories for the quadratic potential, compared to that of the linear case, starts from an orbit with smaller radius and grows to a larger orbit towards the end of inflation.

\section{DISCUSSIONS AND CONCLUSIONS}

Compared to cases with constant running of the spectral index, the perturbation spectrum from periodic mod-

\footnotetext{
${ }^{2}$ For this parameter set, the breakdown of the condition 22 happens at around the end of large-field inflation, hence we disregard the trapping of the inflaton in a local minimum due to the modulations. However, modulations do flatten the potential towards small $\phi$, and extend the period of inflation: The number of efolds obtained from when $\phi_{0} \approx \sqrt{200}$ until the end of inflation is about 50 for a quadratic potential without modulations, but in this case about 57 . Such increase/decrease of e-foldings due to modulations may have interesting consequences, see discussions in Sec. V
}

ulations which we have studied is enhanced at small scales, thus does not delay structure formation 42 . Throughout this work we have assumed that the slow-roll approximations are valid during inflation. If we consider an extreme case where the slow-roll approximations are violated temporally during inflation, a perturbation spectrum with large oscillations at the corresponding scales is realized. Such a spectrum may be used to account for the anomalous suppression at the quadrupole (see [43, 44. and references therein) as well as the missing satellite and/or core-cusp problems [45, 46].

We focused on a case that a sizable running of the spectral index is generated due to the modulations. We may use such a structure to modify the prediction on the spectral index to make an inflation model consistent with observations. One example is chaotic inflation with a quartic coupling, which is strongly disfavored by the WMAP and other observations because the tensor-toscalar ratio is too large for the predicted spectral index. The tension can be relaxed if modulations terminate inflation earlier compared to the case without modulations. Then the inflaton field value when the CMB scale exited the horizon is corrected, modifying predictions on the spectral index and the amplitude of the tensor mode perturbations. Another possibility is that the modulations directly increase the spectral index. However for this to happen, the amplitude of the modulations should decrease towards smaller values of the inflaton, otherwise the inflaton would get trapped by one of the modulations as inflation proceeds.

In this paper, we have explored the possibility of periodic modulations to the inflaton potential giving rise to large running of the spectral index at all scales. Due to the oscillatory modulations, the spectral index $n_{s}-1$ as well as its running $d n_{s} / d \ln k$ switch between positive and negative values. Such behavior results in distinct features in the perturbation spectrum at small scales, which may be probed by future $21 \mathrm{~cm}$ observations. As explicit examples, we have studied inflation models with power-law potentials with superimposed periodic oscillations. We showed that the modulations can produce a running spectral index, while having small impact on the overall inflaton dynamics. In order for the cosmological observables to lie at the central values of the WMAP bounds, modulations with amplitudes of order the GUT scale were considered. Especially in large-field models, as the inflaton travels super-Planckian field ranges, modulations in the potential can show up with scales determined by the microphysics governing inflation. Concrete constructions of such cases in the context of string theory were performed in 21, 22, which realized linear inflaton potentials with superimposed oscillations.

Though we focused on modulations with a large oscillation period encompassing the entire CMB scale, cases with small periods can also have interesting consequences, such as oscillations of the perturbation spectrum within the observed scales [27, 40, 47]. Very small oscillations whose period is sufficiently shorter than one e-fold 
during inflation, can further lead to resonance effects in the power spectrum as well as in the non-Gaussianity, as was studied in [22, 37, 48, 49].

Large running of the spectral index, if confirmed, may suggest the presence of abundant features in the inflaton potential, indicating connections with the microphysics. It would be interesting to examine what we can say about the underlying physics if future observations detect combinations of signals, such as tensor modes and a running spectral index.

\section{Acknowledgments}

We would like to thank Juerg Diemand, Raphael Flauger, Masahiro Kawasaki, Hironao Miyatake, Shinji Mukohyama, and Naoki Yoshida for helpful conversations. The work of T.K. was supported by Grant-in-Aid for JSPS Fellows No. 21.8966. This work was supported by World Premier International Research Center Initiative (WPI Initiative), MEXT, Japan.
[1] A. A. Starobinsky, Phys. Lett. B 91, 99 (1980).

[2] K. Sato, Mon. Not. Roy. Astron. Soc. 195, 467 (1981).

[3] A. H. Guth, Phys. Rev. D 23, 347 (1981).

[4] J. Dunkley et al., arXiv:1009.0866 [astro-ph.CO].

[5] D. Larson et al., arXiv:1001.4635 [astro-ph.CO].

[6] A. A. Starobinsky, JETP Lett. 55, 489 (1992) [Pisma Zh. Eksp. Teor. Fiz. 55, 477 (1992)].

[7] D. J. H. Chung, G. Shiu and M. Trodden, Phys. Rev. D 68, 063501 (2003) arXiv:astro-ph/0305193.

[8] J. M. Cline and L. Hoi, JCAP 0606, 007 (2006) arXiv:astro-ph/0603403.

[9] J. R. Espinosa, arXiv:hep-ph/0605150

[10] M. Joy, V. Sahni and A. A. Starobinsky, Phys. Rev. D 77, 023514 (2008) arXiv:0711.1585 [astro-ph]].

[11] M. Joy, A. Shafieloo, V. Sahni and A. A. Starobinsky, JCAP 0906, 028 (2009) arXiv:0807.3334 [astro-ph]].

[12] M. Kawasaki, M. Yamaguchi and J. Yokoyama, Phys. Rev. D 68, 023508 (2003) arXiv:hep-ph/0304161.

[13] M. Yamaguchi and J. Yokoyama, Phys. Rev. D 68, 123520 (2003) arXiv:hep-ph/0307373.

[14] R. Easther and H. Peiris, JCAP 0609, 010 (2006) arXiv:astro-ph/0604214.

[15] B. Feng, M. z. Li, R. J. Zhang and X. m. Zhang, Phys. Rev. D 68, 103511 (2003) arXiv:astro-ph/0302479.

[16] K. Freese, J. A. Frieman and A. V. Olinto, Phys. Rev. Lett. 65, 3233 (1990).

[17] F. C. Adams, J. R. Bond, K. Freese, J. A. Frieman and A. V. Olinto, Phys. Rev. D 47, 426 (1993) arXiv:hep$\mathrm{ph} / 9207245$.

[18] N. Arkani-Hamed, H. C. Cheng, P. Creminelli and L. Randall, Phys. Rev. Lett. 90, 221302 (2003) arXiv:hep-th/0301218.

[19] N. Arkani-Hamed, H. C. Cheng, P. Creminelli and L. Randall, JCAP 0307, 003 (2003) arXiv:hepth/0302034.

[20] E. Silverstein and A. Westphal, Phys. Rev. D 78, 106003 (2008) arXiv:0803.3085 [hep-th]].

[21] L. McAllister, E. Silverstein and A. Westphal, Phys. Rev. D 82, 046003 (2010) arXiv:0808.0706 [hep-th]].

[22] R. Flauger, L. McAllister, E. Pajer, A. Westphal and G. Xu, JCAP 1006, 009 (2010) arXiv:0907.2916 [hepth]].

[23] S. H. Tye, J. Xu and Y. Zhang, JCAP 0904, 018 (2009) arXiv:0812.1944 [hep-th]].

[24] S. H. Tye and J. Xu, Phys. Lett. B 683, 326 (2010) arXiv:0910.0849 [hep-th]].

[25] A. Lewis and A. Challinor, Phys. Rev. D 76, 083005 (2007) arXiv:astro-ph/0702600.

[26] M. Tegmark and M. Zaldarriaga, Phys. Rev. D 79,
083530 (2009) arXiv:0805.4414 [astro-ph]]

[27] J. Hamann, S. Hannestad, M. S. Sloth and Y. Y. Y. Wong, JCAP 0809, 015 (2008) arXiv:0807.4528 [astro-ph]].

[28] E. D. Stewart and D. H. Lyth, Phys. Lett. B 302, 171 (1993) arXiv:gr-qc/9302019.

[29] J. Garcia-Bellido and D. Wands, Phys. Rev. D 54, 7181 (1996) arXiv:astro-ph/9606047.

[30] W. H. Kinney, Phys. Rev. D 56, 2002 (1997) arXiv:hep$\mathrm{ph} / 9702427$.

[31] A. D. Linde, JHEP 0111, 052 (2001) arXiv:hepth/0110195.

[32] W. H. Kinney, Phys. Rev. D 72, 023515 (2005) arXiv:grqc/0503017.

[33] K. Tzirakis and W. H. Kinney, Phys. Rev. D 75, 123510 (2007) arXiv:astro-ph/0701432.

[34] L. Kofman and S. Mukohyama, Phys. Rev. D 77, 043519 (2008) arXiv:0709.1952 [hep-th]].

[35] T. Kobayashi, S. Mukohyama and B. A. Powell, JCAP 0909, 023 (2009) arXiv:0905.1752 [astro-ph.CO]].

[36] F. Takahashi, Phys. Lett. B 693, 140 (2010) arXiv:1006.2801 [hep-ph]].

[37] X. Chen, R. Easther and E. A. Lim, JCAP 0804, 010 (2008) arXiv:0801.3295 [astro-ph]].

[38] E. Komatsu et al., arXiv:1001.4538 [astro-ph.CO].

[39] E. Komatsu et al. [WMAP Collaboration], Astrophys. J. Suppl. 180, 330 (2009) arXiv:0803.0547 [astro-ph]].

[40] X. Wang, B. Feng, M. Li, X. L. Chen and X. Zhang, Int. J. Mod. Phys. D 14, 1347 (2005) arXiv:astro$\mathrm{ph} / 0209242$.

[41] N. Kaloper and L. Sorbo, Phys. Rev. Lett. 102, 121301 (2009) arXiv:0811.1989 [hep-th]].

[42] N. Yoshida, A. Sokasian, L. Hernquist and V. Springel, Astrophys. J. 598, 73 (2003) arXiv:astro-ph/0305517.

[43] C. L. Bennett et al., arXiv:1001.4758 [astro-ph.CO].

[44] M. Kawasaki and F. Takahashi, Phys. Lett. B 570, 151 (2003) arXiv:hep-ph/0305319.

[45] M. Kamionkowski and A. R. Liddle, Phys. Rev. Lett. 84, 4525 (2000) arXiv:astro-ph/9911103.

[46] J. J. Dalcanton and C. J. Hogan, Astrophys. J. 561, 35 (2001) arXiv:astro-ph/0004381.

[47] C. Pahud, M. Kamionkowski and A. R. Liddle, Phys. Rev. D 79, 083503 (2009) arXiv:0807.0322 [astro-ph]].

[48] S. Hannestad, T. Haugbolle, P. R. Jarnhus and M. S. Sloth, JCAP 1006, 001 (2010) arXiv:0912.3527 [hep-ph]].

[49] R. Flauger and E. Pajer, arXiv:1002.0833 [hep-th]. 